\title{
Microalgal docosahexaenoic acid decreases plasma triacylglycerol in normolipidaemic vegetarians: a randomised trial
}

\author{
Julia Geppert, Veronika Kraft, Hans Demmelmair and Berthold Koletzko* \\ Division of Metabolic Diseases and Nutrition, DR. von Hauner Children's Hospital, Ludwig Maximilians University of Munich, \\ Lindwurmstraße 4, D-80337 Munich, Germany
}

(Received 19 April 2005 - Revised 11 November 2005 - Accepted 6 December 2005)

\begin{abstract}
Triacylglycerol (TG) lowering effects of $n-3$ long-chain PUFA ( $n$-3 LCPUFA) have been repeatedly demonstrated, but studies investigating the individual effects of EPA or DHA on plasma TG and lipoproteins in man are rare. The effects of a new DHA-rich, almost EPA-free microalgae oil (Ulkenia sp.) on plasma lipids and several safety parameters were investigated in a double-blind, placebo-controlled, parallel design intervention study. Normolipidaemic vegetarians (eighty-seven females, twenty-seven males) consumed daily microalgae oil (0.94 g DHA/d) or olive oil (as placebo) for 8 weeks. DHA supplementation decreased plasma TG by $23 \%$ from 1.08 (SEM 0.07) to 0.83 (SEM 0.04) mmol/l $(P<0.001$ ). Absolute TG decreases after DHA supplementation were inversely correlated to baseline TG concentrations $(r-0 \cdot 627, P<0 \cdot 001)$. Plasma total, LDL and HDL cholesterol increased significantly in the DHA group, resulting in lower TG:HDL cholesterol and unchanged LDL:HDL and total cholesterol:HDL cholesterol ratios. The intake of DHA-rich microalgae oil did not result in any physiologically relevant changes of safety and haemostatic factors. In conclusion, DHA-rich oil from microalgae Ulkenia sp. was well tolerated and can be considered a suitable vegetarian source of $n-3$ LCPUFA. Although DHA supplementation improved some CHD risk factors (plasma TG, TG:HDL cholesterol ratio), LDL cholesterol increased. Therefore, the overall effects of this intervention on CHD risk deserve further investigation.
\end{abstract}

Docosahexaenoic acid: Microalgae oil: Plasma lipids: Triacylglycerol

Circulating triacylglycerol (TG) levels in the fasting and postprandial states are associated with the severity and progression of atherosclerosis (Hodis, 1999) and are recognised as independent risk factors for CHD (Hokanson \& Austin, 1996). A meta-analysis by Austin et al. (1998) suggested that after adjustment for HDL cholesterol and other risk factors, each $1 \mathrm{mM}$ increase in TG is associated with a $14 \%$ increase in CHD in men and a $37 \%$ increase in women. Griffin (2001) concluded there is convincing evidence to show that even moderately raised plasma TG $(>1.5 \mathrm{~mm})$, which has a predicted frequency of between 25 and $30 \%$ in middle-aged men and postmenopausal women, confer increased cardiovascular risk in otherwise normal, healthy individuals.

Long-chain $n$-3 PUFA (n-3 LCPUFA) reduce TG concentrations in man (Conquer \& Holub, 1996; Davidson et al. 1997; Grimsgaard et al. 1997; Nelson et al. 1997; Stark \& Holub, 2004; Nestel et al. 2002). In a meta-analysis of sixty-five studies, Harris (1997) concluded that an average dose of $4 \mathrm{~g}$ EPA and DHA per day results in a $25-30 \%$ decrease of fasting TG in both normolipidaemic and hypertriacylglycerolaemic subjects. It has been assumed that the hypotriglyceridaemic effect of $n-3$ LCPUFA is mediated by several mechanisms such as increased hepatic fatty acid oxidation, inhibition of fatty acid and TG synthesis, and reduced assembly and secretion of VLDL TG (Nestel, 2000). Most studies of n-3 LCPUFA have generally used oils containing mixtures of EPA and DHA in the range of $1-5 \mathrm{~g} / \mathrm{d}$, but studies investigating specifically the effects of one $n-3$ LCPUFA on TG and lipoproteins in man are rare.

Studies applying single-cell oil as DHA source, which contain only trace amounts of EPA and other $n-3$ fatty acids, reported a significant reduction in TG concentrations and an increase in HDL cholesterol levels at DHA intakes in the range of $1.25-2.5 \mathrm{~g} / \mathrm{d}$ in normo- or hyperlipidaemic volunteers (Agren et al. 1996; Conquer \& Holub, 1996; Davidson et al. 1997; Nelson et al. 1997), whereas other studies with DHA intakes of $0.7 \mathrm{~g} / \mathrm{d}$ (Theobald et al. 2004) and $0.75-1.5 \mathrm{~g} / \mathrm{d}$ (Conquer \& Holub, 1998) did not find any significant effects on plasma TG and lipoproteins in normolipidaemic subjects.

Few studies have compared the effects of purified EPA and DHA (as ethyl esters), showing inconsistent results. In a study by Rambjor et al. (1996), supplementing normolipidaemic subjects with $3 \mathrm{~g} / \mathrm{d}$ EPA or DHA for 3 weeks, EPA, but not DHA, had a TG-lowering effect. Other studies show that both EPA and DHA lower serum TG concentrations at intakes of 3.0-4.9 g/d (Grimsgaard et al. 1997; Mori et al. 2000; Nestel et al. 2002; Buckley et al. 2004), whereas HDL cholesterol concentrations tend to increase only with DHA supplementation (Rambjor et al. 1996; Grimsgaard et al. 1997; Mori et al. 2000). Future trials will be needed 
to determine minimum effective dosages of EPA and DHA over lengthy periods and to show reduction of CHD by intervention.

The aim of the present study was to investigate the effects of a relatively low dose of DHA $(0.94 \mathrm{~g} / \mathrm{d}$, derived from microalgae Ulkenia sp.) on conventional cardiovascular risk factors (plasma total cholesterol, lipoproteins and TG) and on several safety parameters (e.g. haematology, biochemical markers of liver and cardiac functions, and certain haemostatic risk factors) in normolipidaemic vegetarians. Measures of total cholesterol:HDL cholesterol, LDL cholesterol:HDL cholesterol and TG:HDL cholesterol ratios were of particular interest as by some authors they are considered superior predictors of CHD compared with total and LDL cholesterol levels (Grover et al. 1994, 1995; Kinosian et al. 1994, 1995; Gaziano et al. 1997).

\section{Subjects and methods}

\section{Subjects and study design}

One hundred and fourteen free-living, apparently healthy vegetarians aged 18-43 years (eighty-seven females, twentyseven males) were recruited in the Munich area via posters displayed in health food shops and on the university campus, and through personal contacts. Inclusion criteria included adherence to a vegetarian diet for at least one year (no meat, less than one fish meal per month), age $\geq 18$ years and BMI between 18 and $25 \mathrm{~kg} / \mathrm{m}^{2}$. Exclusion criteria were an intake of medication with known influence on the lipid metabolism during the last 3 months, intake of $n-3$ fatty acid supplements, presence of metabolic, cardiovascular, renal or neurological diseases, or pregnancy and lactation. The study was approved by the ethics committee of the Bavarian Board of Physicians. Written informed consent was obtained from all subjects. Participants received a financial compensation of 200 Euro each for their participation in the study.

The study was conducted between June and November 2003 as a randomised double-blind, placebo-controlled intervention study with two parallel groups. The subjects consumed $2.28 \mathrm{~g}$ daily of either DHA-rich oil from microalgae Ulkenia sp. providing $0.94 \mathrm{~g}$ DHA, or the same amount of olive oil (as placebo) for 8 weeks. They were randomly assigned to one of the intervention groups with stratification for gender. There was no difference between the groups with respect to age, BMI, blood pressure (BP), heart rate, duration of vegetarian diet, proportion of non-smokers and gender (Table 1). Before the first visit, the subjects had to complete a questionnaire, which included a survey on medications, metabolic diseases, CVD, dietary supplements, frequency of fish and egg consumption, and a $3 \mathrm{~d}$ dietary record (two weekdays and one weekend day). During the intervention, the subjects noted side-effects, signs of illness, intake of medication and the number of capsules not consumed. At the end of the intervention period, they recorded their diet again for $3 \mathrm{~d}$. At baseline and after 56-60d of intervention, fasted blood samples were obtained and body weight, height, BP and heart rate were measured. Telephone interviews were performed every 2 weeks of the intervention period to monitor study compliance, side-effects and intercurrent disease. Compliance was
Table 1. Baseline characteristics of the subjects

\begin{tabular}{|c|c|c|c|c|c|}
\hline & \multicolumn{2}{|c|}{$\mathrm{DHA}(n 59)$} & \multicolumn{2}{|c|}{ Placebo (n 55) } & \multirow[b]{2}{*}{$P^{*} \dagger$} \\
\hline & Mean & SD & Mean & SD & \\
\hline $\begin{array}{l}\text { No. of female/male } \\
\text { subjects }\end{array}$ & $44 / 15$ & & $43 / 12$ & & 0.667 \\
\hline Age (years) & $25 \cdot 7$ & $5 \cdot 4$ & $26 \cdot 1$ & $5 \cdot 8$ & 0.902 \\
\hline BMl $\left(\mathrm{kg} / \mathrm{m}^{2}\right)$ & $21 \cdot 4$ & $1 \cdot 8$ & $21 \cdot 2$ & $2 \cdot 0$ & 0.532 \\
\hline \multicolumn{6}{|l|}{ Blood pressure $(\mathrm{mmHg})$} \\
\hline Systolic & 98 & 10 & 96 & 8 & 0.571 \\
\hline Diastolic & 67 & 9 & 67 & 7 & 0.733 \\
\hline Heart rate (beats/min) & 67 & 9 & 67 & 7 & 0.870 \\
\hline $\begin{array}{l}\text { Years on a vegetarian } \\
\text { diet }\end{array}$ & $9 \cdot 8$ & $5 \cdot 5$ & $8 \cdot 8$ & $4 \cdot 8$ & 0.442 \\
\hline Non-smokers (\%) & 75 & & 80 & & 0.512 \\
\hline
\end{tabular}

DHA, docosahexaenoic acid.

*The between-group differences at baseline were analysed using Student's unpaired $t$ test (BMI) or Mann-Whitney $U$ test (age, systolic and diastolic blood pressure, heart rate, years on a vegetarian diet).

†For bivariate tabular analysis the $\chi^{2}$ test was used (gender ratio, number of nonsmokers).

assessed by counting leftover capsules and calculated as the percentage of the prescribed capsules taken.

\section{Study oils}

Each DHA capsule contained $571 \mathrm{mg}$ oil derived from microalgae Ulkenia sp. (Nutrinova ${ }^{\circledR}$ DHA; Nutrinova GmbH, Frankfurt/Main, Germany). Nutrinova ${ }^{\circledR}$ DHA is a highly concentrated, TG-based oil, containing at least $43 \mathrm{~g}$ DHA/100 g total fatty acids (wt \%) and almost no EPA. Matching placebo capsules contained $562 \mathrm{mg}$ olive oil, which is free of $n$-3 LCPUFA. The fatty acid composition of the two study oils as determined during the intervention period is given in Table 2. To ensure that the treatments had a similar antioxidant content, each oil contained $1000 \mathrm{ppm}$ mixed natural tocopherols (equals $2 \cdot 2-2 \cdot 3 \mathrm{mg}$ mixed natural tocopherols per day). The volunteers were required to take one capsule each during breakfast and dinner and two capsules during lunch (a total of four capsules per day). Capsules were stored refrigerated or at room temperature in a dry, dark place.

Table 2. Major fatty acids of microalgae and placebo oil $(\mathrm{g} / 100 \mathrm{~g}$ fatty acids)

\begin{tabular}{lccccc}
\hline & \multicolumn{2}{c}{ Microalgae oil $(n 12)$} & & \multicolumn{2}{c}{ Placebo oil $(n 12)$} \\
\cline { 2 - 3 } \cline { 5 - 6 } & Mean & SD & & Mean & SD \\
\hline SFA & 39.99 & 0.25 & & 14.62 & 0.98 \\
$18: 1 n-9$ & 0.52 & 0.03 & & $75 \cdot 86$ & 0.76 \\
$18: 1 n-7$ & 0.08 & 0.01 & & 2.37 & 0.04 \\
$18: 2 n-6$ & 1.21 & 0.08 & & 5.32 & 0.14 \\
$18: 3 n-6$ & 0.22 & 0.01 & ND & \\
$20: 4 n-6$ & 0.09 & 0.01 & & ND & \\
$22: 5 n-6$ & 9.70 & 0.14 & & ND & \\
$18: 3 n-3$ & 0.11 & 0.01 & & 0.68 & 0.02 \\
$20: 5 n-3$ & 0.29 & 0.01 & & ND & \\
$22: 5 n-3$ & 0.09 & 0.01 & ND & \\
$22: 6 n-3$ & 46.13 & 0.20 & ND & \\
\hline
\end{tabular}

ND, not detected; SFA, saturated fatty acids. 
Measurements, blood sampling and storage

All anthropometric measurements followed standardised procedures. Subjects were weighed before and after the intervention; height was measured only at entry. Seated BP was determined using a stethoscope and a standard sphygmomanometer. Heart rate was measured for $15 \mathrm{~s}$ at the wrist.

Venous blood samples were collected from an antecubital vein of the forearm into EDTA-, citrate- and lithium heparin-containing tubes (Sarstedt, Nümbrecht, Germany) as well as special tubes for platelet function analysis (containing sodium citrate, 1:10) after an overnight fast. For factor VII, von Willebrand factor and PAI-1 analyses, citrated blood, and for vitamin E and fatty acid analyses, EDTA blood was centrifuged at $1000 \mathrm{~g}$ for $7 \mathrm{~min}$ at room temperature within $2 \mathrm{~h}$. The sub-samples of plasma were stored at $-80^{\circ} \mathrm{C}$ and analysed within 12 months of storage; these parameters for each subject were analysed in a single batch at the end of the study. The other biochemical parameters were analysed on the day of blood drawing with routine methods in the clinical chemistry laboratories of the University of Munich hospital.

\section{Analytical methods}

Three-day dietary records were entered into the computer program Prodi version 4.5 LE 2003 (Wissenschaftliche Verlagsgesellschaft $\mathrm{mbH}$, Stuttgart, Germany). Nutrient intake was calculated based on the German Nutrient Data Base BLS, version II.3 (BgVV, Berlin, Germany).

Fatty acids from the two study oils, plasma phospholipids as well as erythrocyte phosphatidylcholine, phosphatidylethanolamine and total lipids were analysed by capillary GLC (Geppert et al. 2005).

TG, total and HDL cholesterol concentrations were determined in lithium heparin plasma by standard enzymatic methods on a Cobas Integra 800 automated sample processor (Roche, Mannheim, Germany) with the appropriate reagent systems. LDL cholesterol concentrations were calculated using the Friedewald formula (Friedewald et al. 1972).

Platelet function analysis was performed within $2 \mathrm{~h}$ of blood collection in citrated whole blood using a Platelet Function Analyser (PFA-100; Dade Behring, Darmstadt, Germany). Membranes pre-coated with collagen/epinephrine or collagen/adenosine diphosphate were used to stimulate platelet aggregation. Prothrombin time, partial thromboplastin time and fibrinogen were measured in citrated plasma using an automated analyser (Amelung Amax CS-190; Trinity Biotech, Darmstadt, Germany) with commercially available kits (Dade Behring, Marburg, Germany). The concentration of d-dimers was determined in citrated plasma on a Roche Hitachi autoanalyser (Roche) with the appropriate test kit. PAI-1 activities were measured using the Coatest PAI-1 assay kit (Chromogenix-Instrumentation Laboratory, Milan, Italy). Factor VII was measured using an ACL 9000 automated analyser (Instrumentation Laboratory, Milan, Italy) and factor VII-deficient plasma (HemosIL, Factor VII-deficient plasma; Instrumentation Laboratory). Von Willebrand factor analysis was performed on a Behring Coagulation System (BCS; Dade Behring) with a commercial test kit (IL Test ${ }^{\mathrm{TM}}$ Von Willebrand Faktor; Instrumentation Laboratory).
Full blood cell counts were performed in EDTA blood on a coulter analyser (Beckmann Coulter Micro Diff II, Krefeld, Germany). Bilirubin, creatinine, $\gamma$-glutamyl transpeptidase, alanine aminotransferase, aspartate aminotransferase, cholinesterase, creatine kinase, lactate dehydrogenase, uric acid, total protein, C-reactive protein and glucose were measured in lithium heparin plasma on a Roche Hitachi 912 automated sample processor (Roche) with the appropriate reagent systems.

For $\alpha$-tocopherol analysis, $50 \mu \mathrm{l}$ internal standard (retinyl acetate in ethanol-butylated hydroxytoluene) and $500 \mu \mathrm{l}$ precipitation solution (Immundiagnostik AG, Bensheim, Germany) were added to $250 \mu \mathrm{l}$ plasma and the tube was vortexed for $15 \mathrm{~s}$. Hexane $(1 \mathrm{ml})$ was added, the tube was vortexed for $30 \mathrm{~s}$ and centrifuged at $1500 \mathrm{~g}$ for $5 \mathrm{~min}$. This step was performed twice. The hexane extracts were combined, evaporated under nitrogen and redissolved in $100 \mu \mathrm{l}$ mobile phase, which consisted of acetonitril-tetrahydrofuran-methanol-ammonium acetate solution (684:220:68:28, by vol.; Hess et al. 1991). The $\alpha$-tocopherol content of the extracts was quantified by HPLC using a RP 18 analytical column (LiChroCART 250-3, LiChrospher 100, RP18 (5 $\mu \mathrm{m})$; Merck, Darmstadt, Germany) and UV-visible detection at $292 \mathrm{~nm}$. Intra- and inter-assay $\mathrm{CV}$ were below $4 \%$.

\section{Statistical methods}

All statistical analyses were done using the Statistical Package for the Social Sciences, version 12.0 (SPSS Inc., Chicago, IL, USA). After checking for the normal distribution of the data, any differences between the groups at baseline or the impact of treatment on the absolute changes (week 8 minus week 0) in each treatment group were tested for significance using Student's unpaired $t$ test for normally distributed variables or the Mann-Whitney $U$ test for variables not normally distributed. Within each of the two groups, changes from 0 to 8 weeks were determined by Student's $t$ test for dependent samples and Wilcoxon non-parametric test, respectively. Correlations between parameters were estimated by computing Pearson's correlation coefficient in the case of normally distributed values and Spearman's Rho correlation coefficient in the case of other distributions, respectively. For bivariate tabular analysis the $\chi^{2}$ test was used. In cases of expected values smaller than 5, a Fisher exact test was used instead. Unless otherwise noted, results are given as mean values with their standard errors. $P<0.05$ was considered significant.

\section{Results}

Two of the 114 subjects recruited into the study dropped out during the intervention period: one subject in the placebo group came down with a renal colic, which was considered unrelated to the dietary supplement, and the contact with one subject of the DHA group was lost. Five individuals in the DHA group and one in the placebo group were excluded from the analyses. The reasons for exclusions were suspected hypertriacylglycerolaemia (basal TG $>2.5 \mathrm{mmol} / 1, n 2$ ), poor compliance with study protocol $\left(\begin{array}{ll}n & 2) \text { and diarrhoea/vomitus }\end{array}\right.$ for more than $6 \mathrm{~d}$ of the intervention period ( $n 2$, one subject from each group). Thus, 106 subjects are included in the present analysis. 


\section{Compliance and side-effects}

The median number of days in the study was 56 (range 56-60 d) in both groups. Compliance as judged by capsule count was 98 (SD 2) \% for the DHA group and 99 (SD 2) \% for the placebo group, with no significant between-group difference. Sideeffects were reported in the DHA group by $11 \%$ and in the placebo group by $8 \%$ of the subjects (Table 3 ) including gastrointestinal upsets (flatulence, pain, diarrhoea, belching) and minor skin reactions (acne). Side-effects were evenly distributed between DHA and placebo group.

\section{Diet, body weight, blood pressure and heart rate}

Baseline intakes of EPA + DHA (median, 5th and 95th percentile in parentheses) were $23(0,121) \mathrm{mg} / \mathrm{d}$ in the DHA group and $23(3,118) \mathrm{mg} / \mathrm{d}$ in the placebo group and did not change in either group during intervention (Geppert et al. 2005).

Body weight, BMI, BP and heart rate did not differ between DHA and placebo group at baseline and changes from baseline were not significantly different between the two groups. An examination of within-group changes demonstrated a slight but significant increase of systolic BP in the placebo group. In the DHA group we observed the same trend, but the change did not reach significance $(P=0.066)$. Body weight and BMI increased significantly by $0.5 \mathrm{~kg}$ or $0.2 \mathrm{~kg} / \mathrm{m}^{2}$ in the placebo group.

\section{Plasma and erythrocyte fatty acids}

Fatty acid compositions of erythrocyte total lipids, phosphatidylcholine and phosphatidylethanolamine as well as plasma phospholipids were not different between groups at baseline and changed negligibly in the placebo group (phospholipid data are shown in Table 4). After DHA supplementation, no change or little increase was observed for saturated fatty acids (16:0 and 18:0). The MUFA 18:1n-9 decreased significantly in all measured fractions relative to baseline. Microalgae oil supplementation resulted in significant increases of $22: 5 n-6$, EPA and DHA levels and significant decreases of $18: 2 n-6,20: 4 n-6$ and $22: 5 n-3$ levels in all measured fractions relative to baseline (Geppert et al. 2005).

\section{Plasma triacylglycerol and lipoproteins}

Measured lipid metabolism parameters were not different between the two groups at study entry and did not change

Table 3. Reported side-effects of the intervention (number and \%)*

\begin{tabular}{lccccr}
\hline & \multicolumn{2}{c}{$\mathrm{DHA}(n 53)$} & & \multicolumn{2}{c}{ Placebo $(n 53)$} \\
\cline { 2 - 3 } & $n$ & & & $n$ & $\%$ \\
\hline Total side-effects & 6 & 11.3 & & 4 & 7.5 \\
Skin reactions & - & & & 1 & 1.9 \\
Flatulence & 3 & 5.7 & & 2 & 3.8 \\
Stomach ache & 2 & 3.8 & & 1 & 1.9 \\
Diarrhoea & - & & & 2 & 3.8 \\
Belching & 2 & 3.8 & & 1 & 1.9 \\
\hline
\end{tabular}

* Side-effects were evenly distributed between DHA and placebo group $(P>0.05)$. in the placebo group (Table 5). A significant $23 \%$ decrease $(P<0.001)$ in plasma TG concentrations from 1.08 to $0.83 \mathrm{mmol} / \mathrm{l}$ was found in the DHA group. Absolute TG changes from baseline were significantly different between the DHA group $(-0.25 \mathrm{mmol} / \mathrm{l}$, mean $)$ and the placebo group $(-0.00 \mathrm{mmol} / \mathrm{l})$. TG changes (absolute and percentage) after DHA supplementation were inversely correlated with TG concentrations at baseline (Fig. 1). After DHA supplementation, observed TG concentrations did not exceed $1.5 \mathrm{mmol} / \mathrm{l}$, whereas after placebo intervention the values were distributed beyond $2.5 \mathrm{mmol} / \mathrm{l}$. The number of subjects with plasma TG levels $>1.5 \mathrm{mmol} / 1$ did not differ between the DHA and placebo group at week $0(17 \%$ v. $15 \%)$, but after the intervention the frequency was significantly lower in the DHA-supplemented group compared to the placebo group ( $0 \%$ v. $21 \%)$.

Plasma total, LDL and HDL cholesterol increased significantly in the DHA group; the changes from baseline in these parameters were significantly different between DHA and placebo group (Table 5). We found a negative correlation between absolute changes in TG and in HDL concentrations after DHA supplementation $(r-0.308, P=0.025)$. In the DHA group, there were no correlations between individual DHA changes in plasma/erythrocyte and changes in plasma TG, total, LDL and HDL cholesterol concentrations (data not shown).

The ratio of TG to $\mathrm{HDL}$ cholesterol was significantly reduced from 0.75 (SEM 0.08) to 0.51 (SEM 0.04) in the DHA group $(P<0.001)$ and remained unchanged in the placebo group; these changes from baseline were significantly different between the two goups. Total cholesterol:HDL cholesterol and LDL cholesterol:HDL cholesterol ratios were not affected by intervention.

\section{Vitamin E, haematology and blood chemistry}

Absolute $\alpha$-tocopherol levels as well as $\alpha$-tocopherol levels adjusted for cholesterol, TG and the sum of cholesterol and TG ( $\alpha$-tocopherol levels divided by the sum of TG and/or total cholesterol) were not different between the two groups at study entry and did not change in the placebo group. There were no changes in $\alpha$-tocopherol levels and lipidadjusted concentrations of $\alpha$-tocopherol after DHA supplementation (Table 5). Cholesterol-adjusted concentrations of $\alpha$-tocopherol decreased and TG-adjusted concentrations of $\alpha$-tocopherol increased significantly after DHA intake; the changes from baseline in these parameters were significantly different between DHA and placebo groups.

The supplementation of DHA-rich oil from microalgae Ulkenia sp. did not result in any physiologically relevant changes in haematology and blood chemistry (data not shown). Incidence of liver ( $\gamma$-glutamyl transpeptidase, alanine aminotransferase, aspartate aminotransferase, cholinesterase) and cardiac enzymes (creatine kinase, lactate dehydrogenase) out of the laboratory's reference range did not differ either at baseline or at week 8 between the DHA and placebo groups.

\section{Discussion}

Our present results show that a supplementation with $0.94 \mathrm{~g} / \mathrm{d}$ DHA for 8 weeks significantly lowered TG in normolipidaemic 
Table 4. Fatty acid composition of plasma phospholipids at weeks 0 and 8 ( $\mathrm{g} / 100 \mathrm{~g}$ fatty acid)*

\begin{tabular}{|c|c|c|c|c|c|c|c|c|c|c|}
\hline & \multicolumn{4}{|c|}{ DHA group ( $n 53)$} & \multicolumn{5}{|c|}{ Placebo group ( $n$ 53) } & \multirow[b]{3}{*}{$P \dagger$} \\
\hline & \multicolumn{2}{|c|}{ Week 0} & \multicolumn{2}{|c|}{ Week 8} & \multirow[b]{2}{*}{$P \dagger$} & \multicolumn{2}{|c|}{ Week 0} & \multicolumn{2}{|c|}{ Week 8} & \\
\hline & Mean & SEM & Mean & SEM & & Mean & SEM & Mean & SEM & \\
\hline $16: 0$ & $28 \cdot 2$ & 0.2 & 28.4 & 0.2 & 0.129 & $28 \cdot 0$ & 0.3 & $27 \cdot 8$ & 0.3 & 0.373 \\
\hline $18: 0$ & $12 \cdot 1$ & 0.2 & $12 \cdot 0$ & 0.2 & 0.679 & $12 \cdot 1$ & 0.2 & $12 \cdot 1$ & 0.2 & 0.980 \\
\hline $18: 1 n-9$ & $10 \cdot 4$ & 0.2 & $9 \cdot 1$ & 0.2 & $<0.001$ & $10 \cdot 3$ & 0.2 & $10 \cdot 5$ & 0.2 & 0.490 \\
\hline $18: 2 n-6$ & $22 \cdot 1$ & 0.4 & $20 \cdot 7$ & 0.4 & $<0.001$ & $23 \cdot 0$ & 0.4 & 22.9 & 0.4 & 0.712 \\
\hline $20: 4 n-6$ & $8 \cdot 9$ & 0.2 & 8.0 & 0.2 & $<0.001$ & 8.9 & 0.2 & $9 \cdot 0$ & 0.2 & 0.674 \\
\hline $22: 5 n-6$ & 0.36 & 0.02 & 0.66 & 0.02 & $<0.001$ & 0.34 & 0.02 & 0.34 & 0.02 & 0.777 \\
\hline $20: 5 n-3$ & 0.58 & 0.03 & 0.77 & 0.03 & $<0.001$ & 0.57 & 0.04 & 0.52 & 0.03 & 0.062 \\
\hline $22: 5 n-3$ & 0.91 & 0.04 & 0.56 & 0.02 & $<0.001$ & 0.85 & 0.04 & 0.85 & 0.04 & 0.865 \\
\hline $22: 6 n-3$ & $2 \cdot 8$ & 0.1 & $7 \cdot 3$ & 0.2 & $<0.001$ & $2 \cdot 6$ & 0.1 & $2 \cdot 5$ & 0.1 & 0.677 \\
\hline
\end{tabular}

* For details of procedures, see pp. 780-781.

†The within-group differences from 0 to 8 weeks were analysed using Student's paired $t$ test (all parameters other than DHA) and Wilcoxon non-parametric test (DHA).

Table 5. Plasma lipids, lipoproteins and $\alpha$-tocopherol at weeks 0 and $8^{\star}$

\begin{tabular}{|c|c|c|c|c|c|c|c|c|c|c|c|}
\hline & \multicolumn{4}{|c|}{ DHA group ( $n$ 53) } & \multirow{2}{*}{$P \dagger$} & \multicolumn{4}{|c|}{ Placebo group ( $n 53$ ) } & \multirow{2}{*}{$P \dagger$} & \multirow{2}{*}{$P \ddagger$} \\
\hline & \multicolumn{2}{|c|}{ Week 0} & \multicolumn{2}{|c|}{ Week 8} & & \multicolumn{2}{|c|}{ Week 0} & \multicolumn{2}{|c|}{ Week 8} & & \\
\hline $\mathrm{TG}(\mathrm{mmol} / \mathrm{l})$ & 1.08 & 0.07 & 0.83 & 0.04 & $<0.001$ & 1.07 & 0.06 & 1.07 & 0.07 & 0.977 & 0.034 \\
\hline Total cholesterol (mmol/l) & 4.58 & 0.13 & $4 \cdot 85$ & 0.14 & 0.001 & $4 \cdot 72$ & 0.13 & 4.69 & 0.12 & 0.623 & 0.004 \\
\hline LDL cholesterol (mmol/l) & $2 \cdot 45$ & $0 \cdot 10$ & $2 \cdot 71$ & 0.11 & $<0.001$ & $2 \cdot 56$ & $0 \cdot 11$ & $2 \cdot 54$ & $0 \cdot 10$ & 0.764 & 0.003 \\
\hline LDL cholesterol:HDL cholesterol & 1.58 & 0.07 & 1.64 & 0.08 & $0 \cdot 180$ & 1.64 & 0.09 & 1.65 & 0.09 & 0.798 & 0.441 \\
\hline Total cholesterol:HDL cholesterol & 2.92 & $0 \cdot 10$ & 2.87 & 0.09 & 0.485 & 2.95 & $0 \cdot 10$ & 2.97 & 0.11 & 0.790 & 0.486 \\
\hline TG:HDL cholesterol & 0.75 & 0.08 & 0.51 & 0.04 & $<0.001$ & 0.69 & 0.05 & 0.70 & 0.06 & 0.733 & 0.022 \\
\hline$\alpha$-Tocopherol ( $\mu \mathrm{mol} / \mathrm{l})$ & $20 \cdot 7$ & 0.5 & $20 \cdot 3$ & 0.5 & 0.193 & $21 \cdot 2$ & 0.6 & $21 \cdot 1$ & 0.6 & 0.761 & 0.510 \\
\hline$\alpha$-Tocopherol:total cholesterol $(\mu \mathrm{mol} / \mathrm{mmol})$ & 4.58 & 0.09 & $4 \cdot 24$ & 0.09 & $<0.001$ & 4.55 & 0.09 & 4.55 & 0.09 & 0.924 & 0.001 \\
\hline$\alpha$-Tocopherol:TG $(\mu \mathrm{mol} / \mathrm{mmol})$ & $22 \cdot 9$ & 1.4 & $27 \cdot 2$ & 1.4 & 0.001 & $23 \cdot 1$ & $1 \cdot 3$ & 22.9 & $1 \cdot 2$ & 0.830 & 0.008 \\
\hline$\alpha$-Tocopherol:(total cholesterol $+\mathrm{TG})(\mu \mathrm{mol} / \mathrm{mmol})$ & $3 \cdot 70$ & 0.08 & 3.61 & 0.08 & 0.143 & 3.72 & 0.07 & 3.72 & 0.08 & 0.931 & 0.281 \\
\hline
\end{tabular}

TG, triacylglycerol.

${ }^{*}$ For details of procedures, see pp. $780-781$.

†The within-group differences from 0 to 8 weeks were analysed using Student's paired $t$ test (all parameters other than TG:HDL cholesterol) and Wilcoxon non-parametric test (TG:HDL cholesterol).

$\ddagger$ The between-group differences in the absolute changes from baseline at week 8 were analysed using Student's unpaired $t$ test (all parameters other than TG and TG:HDL cholesterol) or Mann-Whitney $U$ test (TG and TG:HDL cholesterol).

(basal TG $<2.5 \mathrm{~mm}$ ) subjects. Previous studies with single-cell oil sources of DHA reported a significant reduction in TG concentrations and an increase in HDL cholesterol levels with DHA intakes in the range $1.6-2.4 \mathrm{~g} / \mathrm{d}$ in normolipidaemic volunteers (Agren et al. 1996; Conquer \& Holub, 1996; Nelson et al. 1997), whereas other studies with DHA intakes of $0.7 \mathrm{~g} / \mathrm{d}$ (Theobald et al. 2004) and $0.75-1.5 \mathrm{~g} / \mathrm{d}$ (Conquer \& Holub, 1998) did not find any significant effects on plasma TG and lipoproteins. In persons with combined hyperlipidaemia, significant reductions in TG and increases in LDL and HDL cholesterol concentrations were observed after supplementation with 1.25 or $2.5 \mathrm{~g} / \mathrm{d}$ DHA (Davidson et al. 1997). $n$-3 LCPUFA lower plasma TG concentrations by several mechanisms such as increased hepatic fatty acid oxidation, inhibition of fatty acid and TG synthesis, and depressed assembly and secretion of VLDL TG (Nestel, 2000). The TG-lowering effect of DHA is greater in subjects with higher initial TG concentrations (Harris, 1997). We found a similar relationship in normolipidae- mic subjects, in whom absolute and percentage TG changes were negatively correlated with baseline TG concentrations.

HDL cholesterol concentrations increased after 8 weeks of DHA supplementation. Plasma TG and HDL cholesterol of all subjects at baseline were inversely correlated $(r-0.207$, $P=0.033$ ), and absolute changes in TG correlated inversely with HDL concentrations after DHA supplementation $(r-0 \cdot 308, P=0 \cdot 025)$. The mechanisms by which DHA supplementation increases HDL cholesterol are not known, but may be related to alterations in lipid transfer protein activity (Abbey et al. 1990). A decrease of cholesteryl ester transfer protein activity would reduce the exchange from HDL cholesterol ester and VLDL TG, resulting in larger, more cholesterol-rich HDL cholesterol particles. Indeed, other studies applying purified DHA (3.6 and $4.9 \mathrm{~g} / \mathrm{d}$, respectively) observed a decrease in the apoA1:HDL cholesterol ratio (Grimsgaard et al. 1997; Buckley et al. 2004). Their findings suggest an effect of DHA supplementation on HDL particle size with 
(a)

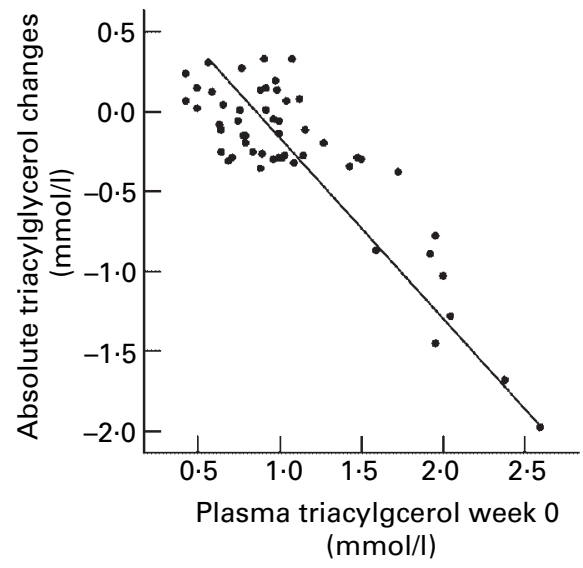

(b)

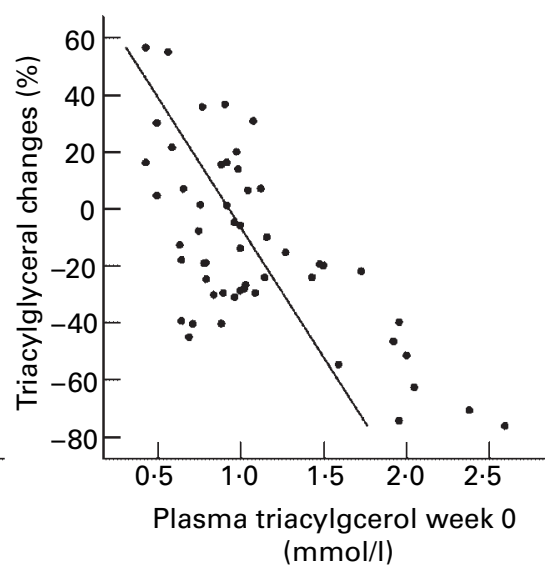

Fig. 1. Correlations between plasma triacylglycerol levels at week 0 and (a) absolute triacylglycerol changes $(n 53, r-0.627, P<0.001)$ and (b) percentage triacylglycerol changes ( $n 53, r-0.534, P<0.001$ ) after 8 weeks of supplementation (Spearman's Rho correlation coefficients). For details of procedures, see pp. $780-781$.

a shift towards the larger, more cholesterol-rich HDL-2 particle. Other investigators observed an increased HDL2:HDL-3 cholesterol ratio (Agren et al. 1996; Rambjor et al. 1996) or increased HDL-2 cholesterol (Mori et al. 2000) after DHA supplementation.

In the present study, total and LDL cholesterol levels increased after DHA intake. Other studies did not detect any significant changes in total cholesterol after supplementation with 0.75-4.9 g/d DHA (Rambjor et al. 1996; Grimsgaard et al. 1997; Conquer \& Holub, 1998; Nestel et al. 2002; Buckley et al. 2004). Inconsistent effects of DHA on LDL cholesterol levels were reported in previous studies; some investigators found a LDL cholesterol-raising effect (Davidson et al. 1997; Mori et al. 2000; Theobald et al. 2004) of DHA or no effect on LDL cholesterol levels (Conquer \& Holub, 1996, 1998; Rambjor et al. 1996; Grimsgaard et al. 1997; Nelson et al. 1997; Nestel et al. 2002; Buckley et al. 2004). The repeatedly observed increase in plasma LDL cholesterol after DHA/fish oil supplementation must be due to either decreased clearance of LDL or increased production of LDL. The limited amount of TG available for packaging into VLDL after supplementation with $n-3$ LCPUFA could result in smaller VLDL particles that contain less TG. Small VLDL are more readily converted to LDL, which may cause increased LDL cholesterol (Griffin, 2001). However, previous studies did not find increases in smaller VLDL subspecies with $n$-3 fatty acid supplementation (Inagaki \& Harris, 1990; Lu et al. 1999). Lu et al. (1999) suggested that $n-3$ fatty acids could enhance the conversion of VLDL to LDL via increased inherent susceptibility of VLDL particles to lipolysis and/or increased in vivo lipolytic activity.

Decreased clearance of LDL might be due to decreases in LDL receptor binding activity or reduced LDL receptor expression. Animal experiments feeding fish oil with DHA to hamsters and primates showed decreased receptor-mediated clearance of LDL cholesterol (Surette et al. 1992; Schectman et al. 1996). Binding of LDL to the LDL receptor decreased because of an altered LDL structure and a reduced affinity of LDL for its receptor. Lindsey et al. (1992) observed an in vitro depression of LDL receptor activity and expression in human hepatoma HepG2 cells after supplementation with $3.6 \mathrm{~g} / \mathrm{d} \mathrm{EPA}$ and $2.9 \mathrm{~g} / \mathrm{d}$ DHA for only 2 weeks. Whether $n-3$ fatty acids alter LDL receptor activity or expression in man is not known, but LDL kinetic studies showed no decrease in fractional catabolic rate relative to a high saturated fat diet (Illingworth et al. 1984) or a vegetable diet (Fisher et al. 1998), providing no evidence for a change in LDL receptor activity in man. Further research is needed to clarify the reasons for the increase in LDL cholesterol with moderate intakes of DHA and the possible consequences for CHD risk in normolipidaemic subjects.

Kinosian et al. $(1994,1995)$ proposed that changes in total cholesterol:HDL cholesterol and LDL:HDL cholesterol ratios are better predictors of risk for CHD than changes in total or LDL cholesterol alone. In our present study, the total cholesterol:HDL cholesterol ratio as well as the LDL:HDL cholesterol remained unchanged in both intervention groups, and the TG:HDL cholesterol ratio was significantly lower after DHA supplementation. The ratio of TG to HDL cholesterol is a predictor of the risk of myocardial infarction (Gaziano et al. 1997). Stark \& Holub (2004) also reported a significant decrease of TG:HDL cholesterol after supplementation with $2.8 \mathrm{~g} / \mathrm{d}$ DHA over 4 weeks in postmenopausal women. Some studies found decreased ratios of total:HDL cholesterol (Conquer \& Holub, 1996; Grimsgaard et al. 1997) and LDL:HDL cholesterol (Conquer \& Holub, 1996) after supplementation with $1.6-3.6 \mathrm{~g} / \mathrm{d}$ DHA or no changes in LDL:HDL or total:HDL cholesterol ratios with DHA intakes of 0.7-2.8 g/d (Stark \& Holub, 2004; Conquer \& Holub, 1998).

In the present study, heart rate and diastolic BP were not affected by DHA supplementation. Similar results were shown previously with DHA intakes of 0.7 and $3.0 \mathrm{~g} / \mathrm{d}$ (Conquer \& Holub, 1998; Nestel et al. 2002). Mori et al. (1999) reported a decrease of systolic and diastolic BP after DHA supplementation in men with otherwise normal BP, whereas Grimsgaard et al. (1998) did not detect a reduction of BP in comparable subjects. Both groups observed a significant influence of DHA on heart rate in healthy men. Very clear effects in respect to $n-3$ LCPUFA on BP control have been observed in hypertensive patients (Knapp \& FitzGerald, 1989; Bonaa et al. 1990; Lungershausen et al. 1994; Toft et al. 1995), but these investigators used 
extremely large amounts of $n-3$ fatty acids ranging from 3.4 to $15 \mathrm{~g} / \mathrm{d}$. In the present study, systolic and diastolic BP at baseline were very low (DHA group, 98/67 mmHg; placebo group, 96/ $67 \mathrm{mmHg}$ ); therefore, a further decrease of BP could not be expected. The observed increase of systolic BP in the placebo group and the same trend in the DHA group may be caused by a systematic change in BP measurement or by environmental influences.

Sanders \& Hinds (1992) reported that plasma $\alpha$-tocopherol concentrations fell below the normal range during a period of fish oil supplementation ( $2.1 \mathrm{~g}$ DHA and $0.8 \mathrm{~g}$ EPA per day), suggesting that fish oil increases the requirement for antioxidants. No significant changes in plasma $\alpha$-tocopherol were evident following fish oil treatment providing $21 \mathrm{mg} / \mathrm{d}$ total tocopherol of which $16 \mathrm{mg}$ was $\alpha$-tocopherol (Leigh-Firbank et al. 2002). In the present study, a total of $2 \cdot 2-2 \cdot 3 \mathrm{mg} / \mathrm{d}$ mixed natural tocopherols was provided by the olive oil and microalgae oil capsules. No changes of $\alpha$-tocopherol levels and lipid-adjusted $\alpha$-tocopherol concentrations were observed after intervention in both groups, suggesting that $2.3 \mathrm{mg}$ mixed natural tocopherols are sufficient to maintain $\alpha$-tocopherol levels at a DHA intake of $0.94 \mathrm{~g} / \mathrm{d}$.

For safety and tolerance evaluation, side-effects were reported and several haematology and biochemistry parameters were analysed. The supplementation of DHA-rich oil from microalgae Ulkenia sp. did not result in any physiologically relevant changes of safety and haemostatic factors. Reported side-effects were equally distributed between DHA and placebo groups.

In conclusion, 8-week supplemenation with DHA-rich microalgae oil was associated with improvements in some CHD risk factors (plasma TG, TG:HDL cholesterol ratio), but others, notably LDL cholesterol, worsened. Therefore, the overall effects of this treatment on CHD risk are unclear and should be further investigated.

\section{Acknowledgements}

We thank Nutrinova (Nutrinova GmbH, Frankfurt/Main, Germany) for providing DHA and placebo capsules and for financial support. We acknowledge the excellent technical assistance of Ismihan Gökce (Department of Internal Medicine, University of Munich) and the team of Anna-Maria Prause (Dr. von Hauner Children's Hospital, University of Munich) and wish to thank the participating subjects for their commitment to this study.

\section{References}

Abbey M, Clifton P, Kestin M, Belling B \& Nestel P (1990) Effect of fish oil on lipoproteins, lecithin:cholesterol acyltransferase, and lipid transfer protein activity in humans. Arteriosclerosis 10, $85-94$.

Agren JJ, Hanninen O, Julkunen A, Fogelholm L, Vidgren H, Schwab U, Pynnonen O \& Uusitupa M (1996) Fish diet, fish oil and docosahexaenoic acid rich oil lower fasting and postprandial plasma lipid levels. Eur J Clin Nutr 50, 765-771.

Austin MA, Hokanson JE \& Edwards KL (1998) Hypertriglyceridemia as a cardiovascular risk factor. Am J Cardiol 81, 7B-12B

Bonaa KH, Bjerve KS, Straume B, Gram IT \& Thelle D (1990) Effect of eicosapentaenoic and docosahexaenoic acids on blood pressure in hypertension. A population-based intervention trial from the Tromso study. N Engl J Med 322, 795-801.

Buckley R, Shewring B, Turner R, Yaqoob P \& Minihane AM (2004) Circulating triacylglycerol and apoE levels in response to EPA and docosahexaenoic acid supplementation in adult human subjects. $\mathrm{Br}$ J Nutr 92, 477-483.

Conquer JA \& Holub BJ (1996) Supplementation with an algae source of docosahexaenoic acid increases (n-3) fatty acid status and alters selected risk factors for heart disease in vegetarian subjects. J Nutr 126, 3032-3039.

Conquer JA \& Holub BJ (1998) Effect of supplementation with different doses of DHA on the levels of circulating DHA as non-esterified fatty acid in subjects of Asian Indian background. J Lipid Res 39, 286-292.

Davidson MH, Maki KC, Kalkowski J, Schaefer EJ, Torri SA \& Drennan KB (1997) Effects of docosahexaenoic acid on serum lipoproteins in patients with combined hyperlipidemia: a randomized, double-blind, placebo-controlled trial. J Am Coll Nutr 16, 236-243.

Fisher WR, Zech LA \& Stacpoole PW (1998) Apolipoprotein B metabolism in hypertriglyceridemic diabetic patients administered either a fish oil- or vegetable oil-enriched diet. J Lipid Res 39, $388-401$.

Friedewald WT, Levy RI \& Fredrickson DS (1972) Estimation of the concentration of low-density lipoprotein cholesterol in plasma, without use of the preparative ultracentrifuge. Clin Chem 18, 499-502.

Gaziano JM, Hennekens CH, O’Donnell CJ, Breslow JL \& Buring JE (1997) Fasting triglycerides, high-density lipoprotein, and risk of myocardial infarction. Circulation 96, 2520-2525.

Geppert J, Kraft V, Demmelmair H \& Koletzko B (2005) Docosahexa enoic acid supplementation in vegetarians effectively increases omega-3 index: a randomized trial. Lipids 40, 870-814.

Griffin BA (2001) The effect of n-3 fatty acids on low density lipoprotein subfractions. Lipids 36, S91-S97.

Grimsgaard S, Bonaa KH, Hansen JB \& Myhre ES (1998) Effects of highly purified eicosapentaenoic acid and docosahexaenoic acid on hemodynamics in humans. Am J Clin Nutr 68, 52-59.

Grimsgaard S, Bonaa KH, Hansen JB \& Nordoy A (1997) Highly purified eicosapentaenoic acid and docosahexaenoic acid in humans have similar triacylglycerol-lowering effects but divergent effects on serum fatty acids. Am J Clin Nutr 66, 649-659.

Grover SA, Coupal L \& Hu XP (1995) Identifying adults at increased risk of coronary disease. How well do the current cholesterol guidelines work? JAMA 274, 801-806.

Grover SA, Palmer CS \& Coupal L (1994) Serum lipid screening to identify high-risk individuals for coronary death. The results of the Lipid Research Clinics prevalence cohort. Arch Intern Med 154, $679-684$.

Harris WS (1997) N-3 fatty acids and serum lipoproteins: human studies. Am J Clin Nutr 65, 1645S-1654S.

Hess D, Keller HE, Oberlin B, Bonfanti R \& Schuep W (1991) Simultaneous determination of retinol, tocopherols, carotenes and lycopene in plasma by means of high-performance liquid chromatography on reversed phase. Int J Vit Nutr Res 61, 232-238.

Hodis HN (1999) Triglyceride-rich lipoprotein remnant particles and risk of atherosclerosis. Circulation 99, 2852-2854.

Hokanson JE \& Austin MA (1996) Plasma triglyceride level is a risk factor for cardiovascular disease independent of high-density lipoprotein cholesterol level: a meta-analysis of population-based prospective studies. J Cardiovasc Risk 3, 213-219.

Illingworth DR, Harris WS \& Connor WE (1984) Inhibition of low density lipoprotein synthesis by dietary omega- 3 fatty acids in humans. Arteriosclerosis 4, 270-275.

Inagaki M \& Harris WS (1990) Changes in lipoprotein composition in hypertriglyceridemic patients taking cholesterol-free fish oil supplements. Atherosclerosis 82, 237-246. 
Kinosian B, Glick H \& Garland G (1994) Cholesterol and coronary heart disease: predicting risks by levels and ratios. Ann Intern Med 121, 641-647.

Kinosian B, Glick H, Preiss L \& Puder KL (1995) Cholesterol and coronary heart disease: predicting risks in men by changes in levels and ratios. J Invest Med 43, 443-450.

Knapp HR \& FitzGerald GA (1989) The antihypertensive effects of fish oil. A controlled study of polyunsaturated fatty acid supplements in essential hypertension. $N$ Engl J Med 320, 1037-1043.

Leigh-Firbank EC, Minihane AM, Leake DS, Wright JW, Murphy MC, Griffin BA \& Williams CM (2002) Eicosapentaenoic acid and docosahexaenoic acid from fish oils: differential associations with lipid responses. Br J Nutr 87, 435-445.

Lindsey S, Pronczuk A \& Hayes KC (1992) Low density lipoprotein from humans supplemented with n-3 fatty acids depresses both LDL receptor activity and LDLr mRNA abundance in HepG2 cells. J Lipid Res 33, 647-658.

Lu G, Windsor SL \& Harris WS (1999) Omega-3 fatty acids alter lipoprotein subfraction distributions and the in vitro conversion of very low density lipoproteins to low density lipoproteins. J Nutr Biochem 10, 151-158.

Lungershausen YK, Abbey M, Nestel PJ \& Howe PRC (1994) Reduction of blood pressure and plasma triglycerides by omega3 fatty acids in treated hypertensives. J Hypertens 12, 1041-1045.

Mori TA, Bao DQ, Burke V, Puddey IB \& Beilin LJ (1999) Docosahexaenoic acid but not eicosapentaenoic acid lowers ambulatory blood pressure and heart rate in humans. Hypertension 34, 253-260.

Mori TA, Burke V, Puddey IB, Watts GF, O’Neal DN, Best JD \& Beilin LJ (2000) Purified eicosapentaenoic and docosahexaenoic acids have differential effects on serum lipids and lipoproteins, LDL particle size, glucose, and insulin in mildly hyperlipidemic men. Am J Clin Nutr 71, 1085-1094.

Nelson GJ, Schmidt PC, Bartolini GL, Kelley DS \& Kyle D (1997) The effect of dietary docosahexaenoic acid on plasma lipoproteins and tissue fatty acid composition in humans. Lipids $\mathbf{3 2}$, $1137-1146$.

Nestel PJ (2000) Fish oil and cardiovascular disease: lipids and arterial function. Am J Clin Nutr 71, 228-231.

Nestel P, Shige H, Pomeroy M, Cehun M, Abbey M \& Raederstorff D (2002) The n-3 fatty acids eicosapentaenoic acid and docosahexaenoic acid increase systemic arterial compliance in humans. Am J Clin Nutr 76, 326-330.

Rambjor GS, Walen AI, Windsor SL \& Harris WS (1996) Eicosapentaenoic acid is primarily responsible for hypotriglyceridemic effect of fish oil in humans. Lipids 31, S45-S49.

Sanders TAB \& Hinds A (1992) The influence of a fish oil high in docosahexaenoic acid on plasma lipoprotein and vitamin E concentrations and haemostatic function in healthy male volunteers. $\mathrm{Br} \mathrm{J}$ Nutr 68, 163-173.

Schectman G, Boerboom LE, Hannah J, Howard BV, Mueller RA \& Kissebah AH (1996) Dietary fish oil decreases low-densitylipoprotein clearance in nonhuman primates. Am J Clin Nutr 64, 215-221.

Stark KD \& Holub BJ (2004) Differential eicosapentaenoic acid elevations and altered cardiovascular disease risk factor responses after supplementation with docosahexaenoic acid in postmenopausal women receiving and not receiving hormone replacement therapy. Am J Clin Nutr 79, 765-773.

Surette ME, Whelan J, Lu GP, Broughton KS \& Kinsella JE (1992) Dependence on dietary cholesterol for n-3 polyunsaturated fatty acid-induced changes in plasma cholesterol in the Syrian hamster. J Lipid Res 33, 263-271.

Theobald HE, Chowienczyk PJ, Whittall R, Humphries SE \& Sanders TAB (2004) LDL cholesterol-raising effect of low-dose docosahexaenoic acid in middle-aged men and women. Am J Clin Nutr 79, 558-563.

Toft I, Bonaa KH, Ingebretsen OC, Nordoy A \& Jenssen T (1995) Effects of n-3 polyunsaturated fatty acids on glucose homeostasis and blood pressure in essential hypertension: a randomized, controlled trial. Ann Intern Med 123, 911-918. 